\title{
Alterações em atributos químicos de um Latossolo pela aplicação de composto de lixo urbano(1)
}

\author{
Fernando Carvalho Oliveira ${ }^{(2)}$, Maria Emilia Mattiazzo(3), Claudio Roberto Marciano ${ }^{(4)}$ \\ e Cassio Hamilton Abreu Junior ${ }^{(5)}$
}

Resumo - Experimento de campo foi conduzido, nos anos agrícolas 1996/97 e 1997/98, para avaliar os efeitos de aplicações sucessivas de composto de lixo urbano sobre os teores de carbono orgânico, condutividade elétrica, $\mathrm{pH}$ e capacidade de troca de cátions (CTC) ao $\mathrm{pH}$ atual de um Latossolo Amarelo distrófico em que foi cultivada cana-de-açúcar. No primeiro ano agrícola, além dos tratamentos calagem + adubação mineral e testemunha, o composto de lixo foi aplicado nas doses de 20, 40 e $60 \mathrm{Mg} \mathrm{ha}^{-1}$ (base seca). No segundo ano, o composto foi reaplicado nas doses de 24,48 e $72 \mathrm{Mg} \mathrm{ha}^{-1}$. Todas as propriedades químicas do solo estudadas aumentaram com as doses do composto, em ambos os anos agrícolas. Os incrementos observados nesses atributos, da primeira para a segunda aplicação, foram significativos, exceto a condutividade elétrica, que, embora tenha aumentado logo após a aplicação do resíduo, não atingiu níveis críticos e teve seus valores reduzidos com o tempo. Acúmulos nos teores de $\mathrm{C}$ orgânico do solo foram diretamente proporcionais às doses de aplicação. $\mathrm{O}$ aumento da CTC do solo foi conseqüência direta dos incrementos nos teores de C orgânico e nos valores de $\mathrm{pH}$.

Termos para indexação: carbono, compostos orgânicos, condutividade elétrica, pH, capacidade de troca de cátions, solo.

\section{Changes on some chemical attributes of a soil due to the application of solid urban waste compost}

Abstract - A field experiment was conducted, during the years of 1996/97 and 1997/98, to evaluate the effects of the successive application of solid urban waste compost on organic carbon, electrical conductivity, $\mathrm{pH}$, and cation exchange capacity (CEC) at field $\mathrm{pH}$ in a Typic Hapludox cropped with sugarcane. In the first year, besides lime + mineral fertilizer and control treatments, the compost was applied at a rate of $0,20,40$, and $60 \mathrm{Mg} \mathrm{ha}^{-1}$ (dry basis). In the second year, the compost was reapplied at a rate of 24,48 , and $72 \mathrm{Mg} \mathrm{ha}^{-1}$. All studied soil properties increased with rate of compost application in both years. Attributes increments from the first to the second application were significant, except for electrical conductivity, which increased soon after the residue application, but did not reach critical levels and decreased along the time. Accumulation of organic $\mathrm{C}$ in soil was direct proportional to compost application rate. The increase of soil CEC was a consequence of the increments on organic $\mathrm{C}$ content and $\mathrm{pH}$ value.

Index terms: carbon, organic compounds, electrical conductivity, $\mathrm{pH}$, cation exchange capacity, soil.

(1) Aceito para publicação em 4 de junho de 2001 .

Extraído da tese de doutorado do primeiro autor apresentada à Escola Superior de Agricultura Luiz de Queiroz (Esalq) Piracicaba, SP. Financiado pela Fapesp.

(2) Biossolo Agricultura \& Ambiente S.C. Ltda., Rua Edu Chaves, 822, São Dimas, CEP 13416-020 Piracicaba, SP E-mail: fernando@biossolidos.com.br

(3) Esalq, Dep. de Ciências Exatas, Caixa Postal 9, CEP 13418-900 Piracicaba, SP. E-mail:mmatiazo@carpa.ciagri.usp.br

(4) Universidade Estadual do Norte Fluminense, Centro de $\mathrm{Ci}$ ência e Tecnologia Agropecuária, Av. Alberto Lamego, 2000, Horto, CEP 28015-620 Campos dos Goytacazes, RJ. E-mail: crmarcia@bol.com.br

(5) Universidade de São Paulo, Centro de Energia Nuclear na Agricultura, Lab. de Fertilidade do Solo, Caixa Postal 96, CEP 13400-970 Piracicaba, SP. E-mail: cahabreu@cena.usp.br

\section{Introdução}

O potencial agronômico do composto de lixo urbano está fundamentado basicamente na elevada concentração de carbono orgânico presente em sua composição. Aumentar o teor de carbono orgânico de um solo pode significar melhorias nas suas propriedades físicas, químicas e biológicas. Além disso, esse resíduo encerra em sua composição apreciáveis quantidades de nutrientes, especialmente $\mathrm{N}, \mathrm{K}$ e $\mathrm{Ca}$ (Xin et al., 1992; Abreu Junior et al., 2000).

A possibilidade desse resíduo de incrementar os teores de carbono orgânico do solo foi verificada em estudos realizados em regiões sob clima temperado. 
Hortenstine \& Rothwell (1972) verificaram aumentos de 41 e $169 \%$ nos teores de C orgânico de um solo degradado por mineração, 24 meses após a aplicação de 35 e $70 \mathrm{Mg} \mathrm{ha}^{-1}$ de composto de lixo, respectivamente. Acréscimo de 31,5\% foi constatado por Bengtson \& Cornette (1973) 28 meses após a aplicação de $44 \mathrm{Mg} \mathrm{ha}^{-1}$ do composto. Giusquiani et al. (1995) também obtiveram acréscimos de 23,40 e $55 \%$ após quatro anos de aplicações anuais sucessivas de 10,30 e $90 \mathrm{Mg} \mathrm{ha}^{-1}$, respectivamente.

Em solos de diferentes regiões do Brasil, Abreu Junior (1999) observou incrementos nos teores de carbono orgânico, em 21 solos ácidos, que variaram de 4 a $35 \%$ e de 8,6 e $34 \%$ em cinco solos alcalinos, 90 dias após aplicação de $60 \mathrm{Mg} \mathrm{ha}^{-1}$ de composto de lixo. Esse autor verificou que existe uma variação no potencial de cada solo para promover a degradação da carga orgânica do resíduo. É possível que em alguns tipos de solos tropicais os efeitos da aplicação do composto de lixo sobre o carbono orgânico do solo sejam efêmeros caso não haja uma freqüência adequada de aplicações.

De acordo com Abreu Junior (1999), a aplicação do composto de lixo em solos ácidos promoveu aumentos nos teores de $\mathrm{K}, \mathrm{Ca}, \mathrm{Mg}$ e Na, em média, de $195 \%, 200 \%, 86 \%$ e $1.200 \%$, respectivamente, e elevação da capacidade de troca catiônica (CTC) em $42 \%$. Nos solos alcalinos, esses efeitos foram menos pronunciados, refletindo, em média, na elevação da CTC em $8,4 \%$.

$\mathrm{O}$ aumento do $\mathrm{pH}$ parece ser um dos principais efeitos da aplicação do composto de lixo em solos ácidos (Xin et al.; 1992; Giusquiani et al., 1995; Abreu Junior et al., 2000). O incremento do pH é diretamente proporcional à capacidade de consumo de prótons, à soma de cátions trocáveis (Wong et al., 1998), e à quantidade e qualidade da matéria orgânica adicionada aos solos pelo material orgânico (Benites \& Mendonça, 1998).

Não obstante as melhorias das propriedades dos solos, tem sido observado o aumento da condutividade elétrica em solos tratados com composto de lixo (Abreu Junior et al., 2000), o que pode causar efeitos fisiológicos irreversíveis às plantas.

Este trabalho teve como objetivo avaliar os efeitos de aplicações sucessivas de doses de composto de lixo urbano sobre os teores de carbono orgânico, condutividade elétrica, $\mathrm{pH}$ e CTC ao $\mathrm{pH}$ atual de um Latossolo Amarelo distrófico cultivado com cana-de-açúcar.

\section{Material e Métodos}

O experimento foi realizado nos anos agrícolas de 1996/97 e 1997/98, em condições de campo, numa área plana de Latossolo Amarelo distrófico pertencente à Estação Experimental do Instituto Agronômico de Campinas, em Piracicaba, SP. O clima, de acordo com a classificação de Köppen, é do tipo Cwa. As principais características químicas e físicas do solo são apresentadas na Tabela 1.

Foram empregadas duas remessas de composto de lixo urbano, obtidas na Usina de Tratamento de Lixo da Cidade de São Jorge, Prefeitura Municipal de Santo André, SP. A caracterização química parcial dos resíduos (Tabela 2) foi realizada de acordo com a metodologia de Eaton et al. (1995).

Estabeleceram-se cinco tratamentos num delineamento em blocos casualizados com quatro repetições, em parcelas experimentais de $100 \mathrm{~m}^{2}$ cultivadas com cana-de-açúcar em ciclo anual. Os tratamentos foram: calagem + adubação mineral (AM); testemunha absoluta (T); composto de lixo nas doses A (CLa), B (CLb) e $\mathrm{C}$ (CLc). $\mathrm{O}$ tratamento AM constou, além da aplicação de $2 \mathrm{Mg} \mathrm{ha}^{-1}$ de calcário dolomítico (PRNT de 90\%) e adubação de plantio em 1996/97, da adubação de cobertura na 1a soqueira, ano agrícola 1997/98 (Spironello et al., 1996). As respectivas doses de composto de lixo (base seca) foram: primeira aplicação, 1996/97, 20 (A), 40 (B) e $60 \mathrm{Mg} \mathrm{ha}^{-1}$ (C), equivalentes a 6,8, 13,7 e $20,5 \mathrm{Mg} \mathrm{ha}^{-1}$ de $\mathrm{C}$ orgânico; segunda aplicação, 1997/98, 24 (A), 48 (B) e $72 \mathrm{Mg} \mathrm{ha}^{-1}$ (C), equivalentes a 6,8, 13,6 e 20,4 Mg ha-1 de C orgânico. As aplicações do resíduo foram feitas na superfície do solo em área total, com distribuição manual nas parcelas e incorporação, na camada de 0-0,2 m, com enxada rotativa. No ano agrícola 1997/98 a incorporação foi realizada nas entrelinhas da cultura.

$\mathrm{Na}$ análise química do solo foram tomadas amostras compostas de terra, de cinco subamostras coletadas nas entrelinhas da cultura dentro da área útil $\left(25,2 \mathrm{~m}^{2}\right.$ centrais $)$ de cada parcela experimental. Na camada 0-0,2 m foram coletadas amostras, dentro de cada ano agrícola, aos 0,30 , $60,90,150,210,270$ e 360 dias após a incorporação do composto de lixo (DAIC). Todas as amostras foram secas em estufa de circulação forçada de ar a $60^{\circ} \mathrm{C}$, destorroadas e passadas por peneira ABNT no 16 (1,19 mm de malha) para a remoção de pequenas raízes. 
Tabela 1. Características químicas e físicas do Latossolo Amarelo distrófico antes da instalação do experimento.

\begin{tabular}{|c|c|c|c|c|c|c|c|c|c|c|c|}
\hline $\begin{array}{l}\text { Profundidade } \\
\text { (m) }\end{array}$ & $\begin{array}{c}\mathrm{pH} \\
\left(\mathrm{CaCl}_{2}\right)\end{array}$ & $\begin{array}{l}\text { C org. } \\
\left(\mathrm{g} \mathrm{kg}^{-1}\right)\end{array}$ & $\begin{array}{c}\mathrm{P} \\
\left(\mathrm{mg} \mathrm{dm}^{-3}\right)\end{array}$ & $\mathrm{K}$ & $\mathrm{Ca}$ & $\mathrm{Mg}$ & $\begin{array}{c}\mathrm{H}+\mathrm{Al} \\
\left(\mathrm{mmol}_{\mathrm{c}} \mathrm{dm}\right.\end{array}$ & Al & SB & $\mathrm{T}$ & $\begin{array}{c}\mathrm{V} \\
(\%)\end{array}$ \\
\hline $0,00-0,20$ & 4,1 & 9,29 & 3 & 0,3 & 11 & 5 & 47 & 10 & 16,3 & 63,3 & 27,7 \\
\hline $0,20-0,40$ & 4,1 & 8,13 & 2 & 0,3 & 14 & 5 & 47 & 10 & 19,3 & 66,3 & 29,1 \\
\hline $0,40-0,60$ & 4,1 & 6,38 & 1 & 0,1 & 11 & 3 & 47 & 12 & 14,1 & 61,1 & 23,1 \\
\hline $\begin{array}{l}\text { Profundidade } \\
\text { (m) }\end{array}$ & $\mathrm{Al}_{2} \mathrm{O}_{3}$ & $\begin{aligned} & \mathrm{Fe}_{2} \mathrm{O}_{3} \\
&-\left(\mathrm{g} \mathrm{kg}^{-1}\right)\end{aligned}$ & $\mathrm{SiO}_{2}$ & \multicolumn{2}{|c|}{ Areia grossa } & \multicolumn{2}{|c|}{$\begin{array}{l}\text { Areia fina } \\
\left(\mathrm{g} \mathrm{kg}^{-1}\right)\end{array}$} & Silte & \multicolumn{2}{|c|}{ Argila } & $\begin{array}{c}\text { Densidade } \\
\left(\mathrm{kg} \mathrm{m}^{-3}\right)\end{array}$ \\
\hline $0,00-0,20$ & 108 & 71,5 & 121 & \multicolumn{2}{|c|}{195} & \multicolumn{2}{|c|}{329} & 141 & \multicolumn{2}{|c|}{335} & 1.316 \\
\hline $0,20-0,40$ & 144 & 85,8 & 148 & \multirow{2}{*}{\multicolumn{2}{|c|}{194}} & & 248 & 116 & \multicolumn{2}{|c|}{442} & 1.292 \\
\hline $0,40-0,60$ & 150 & 88,7 & 155 & & & & 218 & 90 & \multicolumn{2}{|c|}{481} & 1.277 \\
\hline
\end{tabular}

Tabela 2. Composição química parcial das duas remessas de composto de lixo utilizadas no experimento (médias de três amostras compostas retiradas de cada remessa do composto de lixo) $)^{(1)}$.

\begin{tabular}{lrr}
\hline Atributo analisado & 1996 & 1997 \\
\hline $\mathrm{pH}\left(\mathrm{H}_{2} \mathrm{O}, 1: 2,5\right)$ & 4,77 & 8,00 \\
Poder de neutralização $\left(\% \mathrm{CaCO}_{3}\right.$ eq. $)$ & n.r. & 1,66 \\
Carbono orgânico $\left(\mathrm{g} \mathrm{kg}^{-1}\right)$ & 341,99 & 281,63 \\
$\mathrm{~N}$ total $\left(\mathrm{g} \mathrm{kg}^{-1}\right)$ & 10,12 & 12,71 \\
$\mathrm{~N}^{-N^{-}}+\mathrm{N}_{3}^{-} \mathrm{NH}_{4}{ }^{+}\left(\mathrm{g} \mathrm{kg}^{-1}\right)$ & 1,15 & 2,53 \\
Relação C/N & 33,79 & 22,15 \\
$\mathrm{CTC}$ a pH 7,0 $\left(\mathrm{mmol}_{\mathrm{c}} \mathrm{kg}^{-1}\right)$ & 147,96 & 228,78 \\
$\mathrm{P}$ total $\left(\mathrm{g} \mathrm{kg}^{-1}\right)$ & 1,63 & 4,46 \\
$\mathrm{~K}$ total $\left(\mathrm{g} \mathrm{kg}^{-1}\right)$ & 5,28 & 4,90 \\
$\mathrm{Ca}$ total $\left(\mathrm{g} \mathrm{kg}^{-1}\right)$ & 21,26 & 18,40 \\
$\mathrm{Mg}$ total $\left(\mathrm{g} \mathrm{kg}^{-1}\right)$ & 2,66 & 1,60 \\
$\mathrm{~S}$ total $\left(\mathrm{g} \mathrm{kg}^{-1}\right)$ & 3,01 & 3,78 \\
Na total $\left(\mathrm{g} \mathrm{kg}^{-1}\right)$ & 5,46 & 5,20 \\
\hline
\end{tabular}

(1)Exceto o $\mathrm{pH}$, cuja determinação foi feita a partir do material úmido, os demais resultados são expressos com base no material seco; n.r.: determinação não realizada.

Os teores de C orgânico foram determinados, na camada $0-0,2 \mathrm{~m}$, aos $0,30,60,90,150,210,270$ e 360 DAIC, por método volumétrico de dicromatometria de oxirredução. A taxa de degradação da carga orgânica do composto de lixo foi estimada pelo decréscimo do C orgânico presente no solo, através da aplicação de modelo cinético de primeira ordem (Rodella, 1996), aos dados médios de incrementos de carbono $(i \mathrm{C})$. Essa variável expressa o teor de $\mathrm{C}$ orgânico remanescente no solo, devido ao tratamento, e foi obtida ao longo do período estudado, pela diferença entre o teor de C orgânico nas parcelas tratadas com composto de lixo e o teor presente nas testemunhas.

As determinações de condutividade elétrica (CE) e de $\mathrm{pH}$ foram realizadas nas amostras coletadas na camada $0-0,2 \mathrm{~m}$, aos $30,60,90,150,210,270$ e 360 DAIC. Tais determinações foram realizadas em extrato solo-água, na relação $1: 1(\mathrm{p} / \mathrm{v})$, com leitura em condutivímetro com célula de $1 \mathrm{~cm}$. As determinações de $\mathrm{pH}$ foram realizadas em extratos de $\mathrm{CaCl}_{2} 0,01 \mathrm{~mol} \mathrm{~L}$-1, com relação solo-solução $1: 2,5(\mathrm{p} / \mathrm{v})$.

A CTC foi determinada nas amostras coletadas na camada 0-0,2 m, aos 30, 90, 150, 270 e 360 DAIC. Essas determinações foram realizadas pelo método proposto por Gillman \& Sumpter (1986), utilizando soluções não tamponadas.

As análises estatísticas foram realizadas, separadamente, para cada ano agrícola, em esquema com parcelas subdivididas, sendo as parcelas constituídas pelos tratamentos T, CLa, CLb e CLc e as subparcelas, pelas épocas de coleta das amostras de solo. Foi aplicado o teste F e quando constatada interação significativa, as médias foram testadas, dentro de épocas de amostragem e doses, por modelos de regressão polinomial de $1 \stackrel{0}{\text { e }} 2^{2}$ o grau. Para a variável $i \mathrm{C}$, verificando-se efeitos significativos das causas de variação, foram realizados ajustes de funções exponenciais do tipo $\mathrm{y}=\mathrm{ae}^{-\mathrm{bx}}$. Procedeu-se à análise de correlação linear simples entre valores de $\mathrm{C}$ orgânico, $\mathrm{pHe}$ CTC. Os dados obtidos no tratamento AM foram usados exclusivamente nas análises de correlação entre CTC e pH.

\section{Resultados e Discussão}

Os teores de C orgânico do solo apresentaram, em todas as épocas de amostragem, nos dois anos agrícolas, aumentos lineares em função das doses aplicadas do composto de lixo (Tabela 3 ). Tomando-se como base o tratamento T, verificou-se que aos 360 DAIC, ano agrícola 1996/97, houve aumentos de 8,16 e $22 \%$ nos teores de carbono devido à aplicação do composto em CLa, CLb e CLc, respectivamente. Aos 360 DAIC da segunda aplicação constataram-se aumentos de 18, 29 e 55\% para CLa, CLb e CLc, respectivamente, ou seja, houve um acúmulo de carbono de cerca de 17, 19 e 36\% em relação aos teores observados, nos mesmos tratamentos, no final do ano anterior. 
O composto de lixo aumentou significativamente os teores de carbono do solo, sugerindo a possibilidade de incrementos crescentes em função de aplicações sucessivas do resíduo. Resultados semelhantes foram obtidos por Hortenstine \& Rothwell (1972), Bengtson \& Cornette (1973), Giusquiani et al. (1995) e Abreu Junior (1999).

Os teores de C orgânico foram influenciados significativamente pela interação entre tratamentos e épocas de amostragem do solo. Esperava-se que esta interação fosse devida ao decréscimo dos teores de carbono em função do tempo ocorrido da aplicação, o que foi verdadeiro apenas para CLb e CLc, no ano agrícola 1997/98. No entanto, observou-se que os teores de $\mathrm{C}$ orgânico em $\mathrm{T}$ aumentaram ao longo das épocas de amostragem do solo (Tabela 3). Esses acréscimos foram mais expressivos no primeiro ano agrícola.

O teor de C orgânico original do solo, antes da instalação do experimento, era de 9,29 $\mathrm{g} \mathrm{kg}^{-1}$ (Tabela 1). O seu decréscimo no tempo zero do ano agrícola 1996/97 é justificado pelas operações de preparo do solo antes da aplicação do composto. Tais operações, que constaram de subsolagem, uma aração e uma gradagem, promoveram uma inversão de camadas, aflorando o solo da camada abaixo de $0,2 \mathrm{~m}$, com menor teor de C orgânico (Tabela 1). Outra possibilidade é o estímulo à degradação de parte do $\mathrm{C}$ orgânico nativo do solo pela aeração proporcionada por tais operações. Os teores de C orgânico de T retornaram aos seus valores originais somente 24 meses após o preparo inicial do solo (Tabela 3).

A análise de variância do incremento de carbono (iC), nos dois anos agrícolas, revelou efeitos significativos de doses e épocas de amostragem, e não houve interação entre estes fatores. Desta forma, obteve-se um comportamento médio válido para as três doses do resíduo.

Nos dois anos agrícolas, o decréscimo do C orgânico aplicado ao solo através do composto ajustou-se a um modelo de cinética de primeira ordem (Figura 1). Com base neste modelo matemático, pode-se inferir que o composto de lixo apresentou uma fase inicial de degradação mais rápida seguida por outras de estabilização progressiva. Levi-Minzi et al. (1990) e Barreto (1995) observaram este mesmo comportamento para composto de lixo,

Tabela 3. Teores de carbono orgânico do solo $\left(\mathrm{g} \mathrm{kg}^{-1}\right)$ determinados nas diversas épocas de amostragem, nos anos agrícolas 1996/97 e 1997/98.

\begin{tabular}{|c|c|c|c|c|c|c|}
\hline \multirow[t]{2}{*}{$\mathrm{DAIC}^{(1)}$} & \multicolumn{4}{|c|}{ Dose de composto de lixo $\left(\mathrm{Mg} \mathrm{ha}^{-1}\right)^{(2)}$} & \multicolumn{2}{|c|}{ Coeficiente de determinação da regressão } \\
\hline & $\mathrm{T}$ & $\mathrm{CLa}$ & CLb & CLc & Linear & Quadrática \\
\hline & \multicolumn{6}{|c|}{$1996 / 97$} \\
\hline 0 & 7,16 & 9,44 & 10,96 & 12,20 & $0,98^{* *}$ & ns \\
\hline 30 & 7,09 & 8,68 & 10,00 & 10,39 & $0,94^{* *}$ & ns \\
\hline 60 & 6,94 & 8,13 & 8,98 & 9,51 & $0,97^{* *}$ & ns \\
\hline 90 & 7,58 & 9,02 & 9,97 & 11,18 & $0,99^{* *}$ & ns \\
\hline 150 & 8,74 & 9,76 & 10,93 & 11,87 & $0,99^{* * *}$ & ns \\
\hline 210 & 8,39 & 9,01 & 9,78 & 11,18 & $0,96^{* * *}$ & ns \\
\hline 270 & 8,86 & 9,63 & 10,18 & 10,73 & $0,99^{*}$ & ns \\
\hline 360 & 8,86 & 9,59 & 10,29 & 10,78 & $0,99^{*}$ & ns \\
\hline Regressão linear & $0,79^{* * *}$ & $0,27^{* *}$ & ns & ns & - & - \\
\hline \multirow[t]{2}{*}{ Regressão quadrática } & $0,85^{*}$ & $\mathrm{~ns}$ & ns & $\mathrm{ns}$ & - & - \\
\hline & \multicolumn{6}{|c|}{ 1997/98 } \\
\hline 0 & 8,57 & 11,50 & 13,68 & 16,16 & $0,99^{* *}$ & ns \\
\hline 30 & 8,86 & 11,90 & 13,43 & 15,00 & $0,97^{* *}$ & ns \\
\hline 60 & 8,72 & 11,21 & 13,68 & 14,99 & $0,98^{* * *}$ & ns \\
\hline 90 & 8,87 & 11,51 & 12,53 & 15,43 & $0,97^{* *}$ & ns \\
\hline 150 & 8,98 & 10,75 & 11,83 & 15,75 & $0,93^{* *}$ & ns \\
\hline 210 & 8,99 & 11,06 & 12,29 & 15,51 & $0,97^{* * *}$ & ns \\
\hline 270 & 9,42 & 11,37 & 12,46 & 14,82 & $0,98^{* *}$ & ns \\
\hline 360 & 9,48 & 11,23 & 12,22 & 14,70 & $0,97^{* * *}$ & ns \\
\hline Regressão linear & $0,87^{*}$ & ns & $0,54^{*+1}$ & $0,32^{*}$ & - & - \\
\hline Regressão quadrática & ns & ns & $0,75^{*}$ & ns & - & - \\
\hline
\end{tabular}

${ }^{(1)}$ Dias após a incorporação do composto de lixo. ${ }^{(2)} \mathrm{T}=0, \mathrm{CLa}=20, \mathrm{CLb}=40, \mathrm{CLc}=60 \mathrm{Mg} \mathrm{ha}^{-1} \mathrm{em} \mathrm{1996/97} \mathrm{e} \mathrm{T}=0, \mathrm{CLa}=24, \mathrm{CLb}=48$, $\mathrm{CLc}=72 \mathrm{Mg} \mathrm{ha}^{-1}$ em 1997/98. ns Não-significativo. ${ }^{*} \mathrm{e}^{* *}$ Significativo a 5 e a $1 \%$ de probabilidade pelo teste $\mathrm{F}$, respectivamente. 
em ensaios de respirometria conduzidos em condições de laboratório.

Estimou-se a taxa média de decréscimo do carbono em $66 \%$ no ano agrícola $1996 / 97$, valor que pode ser considerado como estimativa da taxa de degradação do composto de lixo. Neste ano, o revolvimento do solo pelas operações de cultivo mecânico podem ter estimulado a degradação do C orgânico. Em 1997/98, estimou-se a taxa média de decréscimo em 37\%, entretanto, não é possível afirmar que houve queda na taxa de degradação do composto, pois nesta foi incluído o carbono recalcitrante acumulado no solo devido à primeira aplicação.

A degradação do composto de lixo em solos é reduzida drasticamente à medida que aumenta seu grau de maturação (Bernal et al., 1998; Pascual et al., 1998). Considerando-se que a remessa do composto de lixo utilizada em 1997/98 apresentava um grau de maturação mais avançado do que o composto aplicado em 1996/97, como mostram os valores de $\mathrm{pH}$ e relações $\mathrm{C} / \mathrm{N}$ na Tabela 2, é possível que isto tenha contribuído para a menor taxa de decréscimo observada após a segunda aplicação. Além do mais, nenhuma operação mecânica que revolvesse o solo foi executada neste ano, após a incorporação do resíduo.

Considerando-se as taxas e a freqüência de aplicações do resíduo, evidenciou-se que parte do $\mathrm{C}$ orgânico presente no composto de lixo foi resistente à degradação. Estes resultados são concordantes com estudos realizados em condições controladas de laboratório, os quais evidenciaram a relativa resistência à degradação, no solo, da carga orgânica de compostos de lixo (Levi-Minzi et al., 1990; Barreto, 1995).

O composto de lixo aumentou linearmente a condutividade elétrica (CE) do solo nos tratamentos CLa, CLb e CLc, em ambos os anos agrícolas, até aos 210 DAIC no ano agrícola 1996/97 e até aos 270 DAIC no ano seguinte (Tabela 4). Pequenas diferenças no comportamento da CE, entre épocas e anos agrícolas, foram relacionadas à dinâmica da degradação da carga orgânica do composto e principalmente à distribuição de chuvas durante o período experimental. Efeitos semelhantes foram verificados por Benites \& Mendonça (1998).

Os maiores valores de CE foram observados aos 30 DAIC em ambos os anos agrícolas. No primeiro ano, o acréscimo na condutividade elétrica devido à CLc foi de 339\% em relação a T; em 1997/98, tal acréscimo foi da ordem de $336 \%$. Hortenstine \& Rothwell (1972, 1973) e Abreu Junior (1999) também não encontraram efeitos salinos prejudiciais às culturas de aveia, sorgo granífero e arroz, respectivamente.

Tendo em vista a composição química do composto de lixo (Tabela 2), é provável que os aumentos

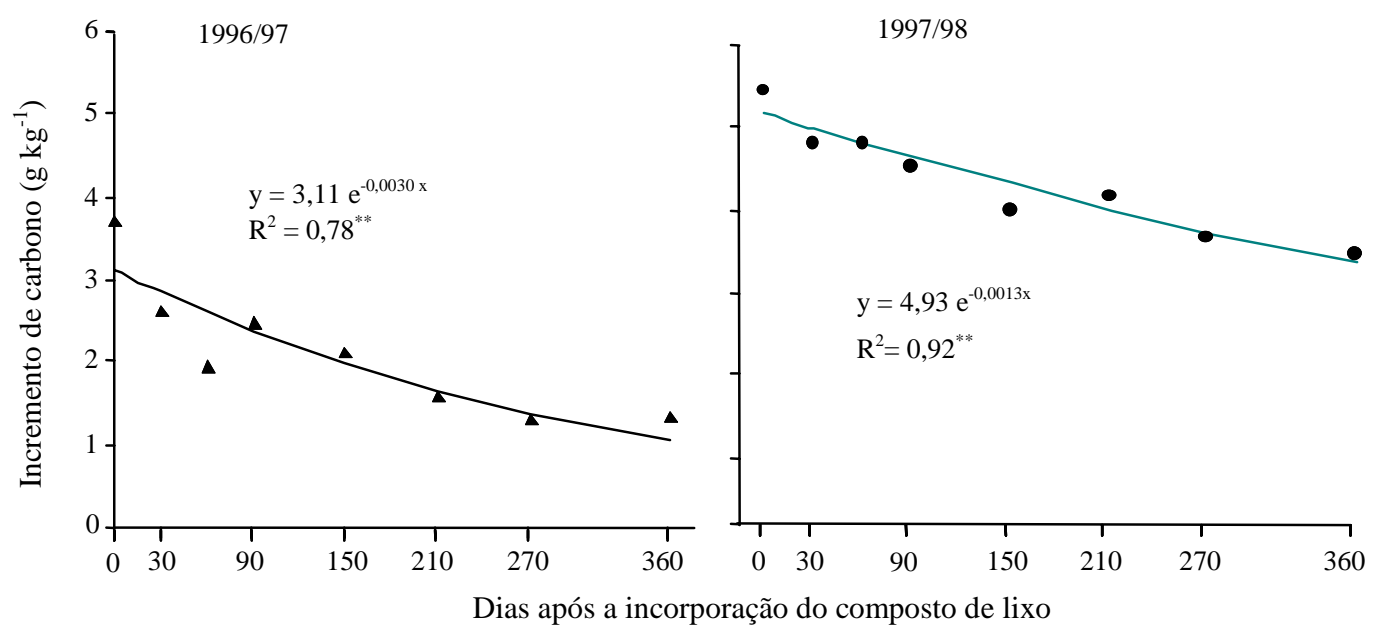

Figura 1. Decréscimo do carbono orgânico adicionado ao solo via composto de lixo, nos anos agrícolas 1996/97 e 1997/98, ajustado a um modelo de cinética de primeira ordem. ${ }^{* *}$ Significativo a $1 \%$ pelo teste $\mathrm{F}$. 
na $\mathrm{CE}$ do solo foram devidos à degradação da matéria orgânica do resíduo e a conseqüente solubilização predominante de íons $\mathrm{NH}_{4}{ }^{+}, \mathrm{NO}_{3}{ }^{-}, \mathrm{Ca}^{2+}, \mathrm{Na}^{+}, \mathrm{K}^{+}$, $\mathrm{SO}_{4}{ }^{2-}$ e $\mathrm{Mg}^{2+}$.

A CE apresentou, nos dois anos agrícolas, dentro das doses de composto de lixo, significativa redução à medida em que aumentou o tempo de incorporação do resíduo (Tabela 4). O decréscimo na concentração salina da superfície do solo foi devido à lavagem de íons solúveis ou em suspensão, proporcionada pelo movimento descendente da água das precipitações pluviais (Oliveira, 2000). Estas observações são semelhantes àquelas feitas por Harding et al. (1985) em solos tratados com elevadas doses de lodo de esgoto.

Diante do comportamento da CE do solo no decorrer do experimento e da ordem de grandeza alcançada pelos seus valores, acredita-se que nas taxas e freqüência em que foram aplicados o composto de lixo, não serão observados efeitos salinos pelo menos a médio prazo. No entanto, como existem variações nesses efeitos em função do solo (Abreu Junior et al., 2000) e do regime pluviométrico de cada região, é recomendável que a aplicação de composto de lixo em solos seja monitorada com relação a esta variável.
Em 1996/97, em todas as épocas de amostragem do solo, o composto proporcionou aumentos lineares no $\mathrm{pH}$ do solo, exceto aos 270 DAIC (Tabela 5). No ano seguinte, as doses de aplicação apresentaram efeitos quadráticos sobre o $\mathrm{pH}$, exceto aos 30 , 90 e 150 DAIC. No primeiro ano agrícola, o pH do solo apresentou acréscimos médios, em relação à $\mathrm{T}$, de 0,5, 0,6 e 1,1 unidades, respectivamente para CLa, CLb e CLc. Em 1997/98, após a reaplicação do composto, esses acréscimos foram de 1,3, 1,6 e 2,1 unidades, respectivamente. Nos tratamentos CLa, CLb e CLc, os acréscimos de $\mathrm{pH}$ em decorrência da segunda aplicação foram de 0,8, 1,0 e 1,1 unidades. Assim, verificou-se que a reaplicação do resíduo proporcionou aumentos compatíveis com aqueles do primeiro ano, o que sugere um efeito acumulativo sobre o $\mathrm{pH}$, por ocasião de aplicações sucessivas do composto de lixo.

O poder de neutralização exercido pelo composto de lixo está relacionado com o comportamento de sua matéria orgânica no solo. Hoyt \& Turner (1975) atribuíram a elevação do $\mathrm{pH}$ de um solo ácido à ação complexante de substâncias orgânicas derivadas da decomposição de resíduos de alfafa, sobre o alumínio presente na solução do solo. Mattiazzo \& Glória

Tabela 4. Condutividade elétrica do solo $\left(\mu \mathrm{S} \mathrm{cm}^{-1}\right)$ determinada na camada de 0-0,2 $\mathrm{m}$, nas diversas épocas de amostragem, nos anos agrícolas 1996/97 e 1997/98.

\begin{tabular}{|c|c|c|c|c|c|c|}
\hline \multirow[t]{2}{*}{$\mathrm{DAIC}^{(1)}$} & \multicolumn{4}{|c|}{ Dose de composto de lixo $\left(\mathrm{Mg} \mathrm{ha}^{-1}\right)^{(2)}$} & \multicolumn{2}{|c|}{ Coeficiente de determinação de regressão } \\
\hline & $\mathrm{T}$ & $\mathrm{CLa}$ & CLb & CLc & Linear & Quadrática \\
\hline & \multicolumn{6}{|c|}{$1996 / 97$} \\
\hline 30 & 101,6 & 152,1 & 426,3 & 445,8 & $0,88^{* *}$ & $\mathrm{~ns}$ \\
\hline 60 & 79,4 & 138,8 & 149,0 & 252,5 & $0,90^{* *}$ & ns \\
\hline 90 & 115,4 & 203,9 & 289,8 & 331,2 & $0,98^{* *}$ & ns \\
\hline 150 & 107,0 & 152,0 & 165,9 & 282,8 & $0,87^{* *}$ & ns \\
\hline 210 & 116,9 & 150,7 & 191,1 & 211,5 & $0,99^{*}$ & $\mathrm{~ns}$ \\
\hline 270 & 62,9 & 86,1 & 104,7 & 115,3 & ns & ns \\
\hline 360 & 73,9 & 110,0 & 113,8 & 138,9 & ns & ns \\
\hline Regressão linear & ns & $0,43^{*}$ & $0,50^{* *}$ & $0,75^{* *}$ & - & - \\
\hline \multirow[t]{2}{*}{ Regressão quadrática } & $\mathrm{ns}$ & ns & $0,59^{* *}$ & $0,79^{*}$ & - & - \\
\hline & \multicolumn{6}{|c|}{$1997 / 98$} \\
\hline 30 & 127,3 & 202,8 & 301,8 & 401,8 & $0,99^{* *}$ & ns \\
\hline 60 & 79,7 & 161,1 & 300,5 & 239,8 & $0,70^{* *}$ & $0,88^{*}$ \\
\hline 90 & 89,8 & 199,0 & 281,2 & 392,5 & $0,99^{* *}$ & ns \\
\hline 150 & 92,2 & 199,0 & 242,0 & 401,6 & $0,95^{* *}$ & $\mathrm{~ns}$ \\
\hline 210 & 61,8 & 125,1 & 233,9 & 245,8 & $0,93^{* *}$ & ns \\
\hline 270 & 80,0 & 162,2 & 184,0 & 237,1 & $0,95^{* *}$ & $\mathrm{~ns}$ \\
\hline 360 & 70,8 & 141,6 & 143,6 & 139,8 & ns & ns \\
\hline Regressão linear & ns & $0,43^{* * *}$ & $0,98^{* *}$ & $0,56^{* *}$ & - & - \\
\hline Regressão quadrática & ns & ns & $\mathrm{ns}$ & $0,62^{* *}$ & - & - \\
\hline
\end{tabular}

${ }^{(1)}$ Dias após a incorporação do composto de lixo. ${ }^{(2)} \mathrm{T}=0, \mathrm{CLa}=20, \mathrm{CLb}=40, \mathrm{CLc}=60 \mathrm{Mg} \mathrm{ha}^{-1}$ em 1996/97 e T $=0, \mathrm{CLa}=24, \mathrm{CLb}=48$, CLc $=72 \mathrm{Mg} \mathrm{ha}^{-1}$ em 1997/98. ns Não-significativo. ${ }^{*} \mathrm{e}^{* *}$ Significativo a 5 e a $1 \%$ de probabilidade pelo teste $\mathrm{F}$, respectivamente. 
(1987) explicaram, num estudo com vinhaça, que esses efeitos são devidos à produção de íons $\mathrm{OH}^{-}$quando o oxigênio da solução do solo atua como receptor final de elétrons derivados da oxidação microbiana do C orgânico do resíduo. Conforme Hue (1992), resíduos orgânicos geram íons $\mathrm{OH}^{-}$no solo através de dois processos: pela troca de ligantes entre ânions orgânicos (p.e. tartarato e ftalato) e terminais hidroxilas de óxidos de $\mathrm{Fe}$ e $\mathrm{Al}$; pela redução de óxidos de $\mathrm{Fe}$ e $\mathrm{Al}$ (predominantemente goethita), que deve ocorrer em ambiente rico em elétrons derivados da rápida oxidação do $\mathrm{C}$ orgânico dos resíduos. Recentemente, Wong et al. (1998) observaram que a troca de $\mathrm{H}^{+}$entre o sistema tampão do solo e o da matéria orgânica do composto de lixo seria o principal mecanismo para a elevação do $\mathrm{pH}$ dos solos tratados.

Aumentos no valor $\mathrm{pH}$ de solos em decorrência da aplicação de composto de lixo foram observados por Hortenstine \& Rothwell (1972), Bengtson \& Cornette (1973), Trindade et al. (1996) e Abreu Junior et al. (2000).

Os valores de $\mathrm{pH}$, dentro de cada dose aplicada, no decorrer das várias épocas de amostragem do solo, diminuíram ao longo de 1996/97 (Tabela 5). Porém, no ano seguinte, tais decréscimos não foram observados para nenhum tratamento com composto, ao contrário, observou-se ligeiro aumento do $\mathrm{pH}$ no decorrer desse ano para CLb. A diminuição do $\mathrm{pH}$ em 1996/97 pode ser explicada pela maior taxa de decréscimo do $\mathrm{C}$ orgânico associado aos processos naturais de acidificação do solo. No ano seguinte, os maiores teores de $\mathrm{C}$ orgânico do solo somados a sua menor taxa de decréscimo, provavelmente conferiram ao solo um maior efeito tampão. Harding et al. (1985) também observaram que a aplicação de lodo de esgoto foi capaz de aumentar e manter o pH do solo até cinco anos depois, efeito atribuído ao poder de tamponamento proporcionado pela lenta e contínua degradação de sua matéria orgânica.

$\mathrm{Na}$ análise de variância da capacidade de troca de cátions (CTC), verificou-se, nos dois anos agrícolas, efeitos significativos de doses de composto de lixo e épocas de amostragem do solo, porém não houve interação entre estes fatores. O aumento da CTC em função das doses do resíduo seguiu, dentro de todas as amostragens de 1996/97, um comportamento linear $\left(\mathrm{CTC}=32,4+0,12 \times C L, \mathrm{R}^{2}=0,95, \mathrm{p}<0,01\right)$ ao passo que para $1997 / 98$, tal comportamento foi quadrático $\left(\mathrm{CTC}=31,4+0,35 \mathrm{xCL}-0,003 \mathrm{xCL}^{2}\right.$, $\left.\mathrm{R}^{2}=0,99, \mathrm{p}<0,01\right)$.

Tabela 5. Valores de $\mathrm{pH}$ do solo determinados nas diversas épocas de amostragem, dentro dos anos agrícolas 1996/97 e 1997/98.

\begin{tabular}{|c|c|c|c|c|c|c|}
\hline \multirow[t]{2}{*}{ DAIC $^{(1)}$} & \multicolumn{4}{|c|}{ Dose de composto de lixo $\left(\mathrm{Mg} \mathrm{ha}^{-1}\right)$} & \multicolumn{2}{|c|}{ Coeficiente de determinação da regressão } \\
\hline & $\mathrm{T}$ & $\mathrm{CLa}$ & CLb & CLc & Linear & Quadrática \\
\hline & \multicolumn{6}{|c|}{$1996 / 97$} \\
\hline 30 & 4,3 & 4,7 & 5,1 & 5,5 & $0,99^{* *}$ & ns \\
\hline 60 & 4,2 & 4,6 & 5,0 & 5,2 & $0,99^{* *}$ & ns \\
\hline 90 & 4,2 & 5,0 & 5,1 & 5,6 & $0,91^{* *}$ & ns \\
\hline 150 & 4,3 & 4,7 & 4,7 & 5,0 & $0,92 *$ & ns \\
\hline 210 & 4,2 & 4,6 & 4,7 & 5,2 & $0,94^{* *}$ & ns \\
\hline 270 & 4,2 & 4,6 & 4,4 & 5,0 & $0,69^{*}$ & $0,99^{* *}$ \\
\hline 360 & 4,1 & 4,5 & 4,5 & 5,0 & $0,88^{* * *}$ & ns \\
\hline Regressão linear & ns & $0,27^{*}$ & $0,82^{* *}$ & $0,58^{* *}$ & - & - \\
\hline \multirow[t]{2}{*}{ Regressão Quadrática } & $\mathrm{ns}$ & ns & $0,88^{*}$ & ns & - & - \\
\hline & \multicolumn{6}{|c|}{$1997 / 98$} \\
\hline 30 & 4,3 & 5,7 & 5,5 & 6,5 & $0,81^{* *}$ & ns \\
\hline 60 & 4,3 & 5,3 & 5,9 & 5,8 & $0,77^{* *}$ & $0,99^{* * *}$ \\
\hline 90 & 4,2 & 5,3 & 5,5 & 6,2 & $0,93^{* *}$ & ns \\
\hline 150 & 4,2 & 5,4 & 5,6 & 6,4 & $0,93^{* *}$ & ns \\
\hline 210 & 4,2 & 5,7 & 5,9 & 6,3 & $0,86^{* *}$ & $0,96^{* *}$ \\
\hline 270 & 4,2 & 5,6 & 5,8 & 6,2 & $0,86^{* *}$ & $0,95^{*}$ \\
\hline 360 & 4,1 & 5,7 & 6,1 & 6,4 & $0,85^{* *}$ & $0,98^{* * *}$ \\
\hline Regressão linear & $\mathrm{ns}$ & $\mathrm{ns}$ & $0,44^{* *}$ & $\mathrm{~ns}$ & - & - \\
\hline Regressão quadrática & ns & $\mathrm{ns}$ & ns & ns & - & - \\
\hline
\end{tabular}

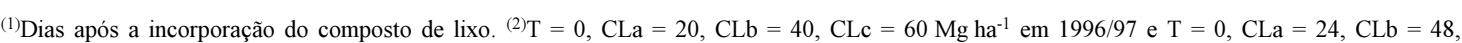

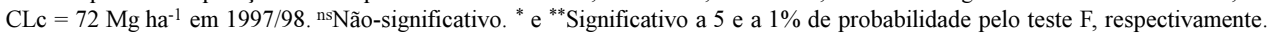


Em 1996/97, foi verificado, através da análise de regressão, um comportamento quadrático decrescente nos valores de CTC em função das épocas de amostragem do solo (EAs) $(\mathrm{CTC}=36,1+0,01 \times \mathrm{xEAs}$ $\left.0,00004 \times \mathrm{xAs}^{2}, \mathrm{R}^{2}=0,85, \mathrm{p}<0,01\right)$. Os valores de CTC, máximos e mínimos, encontrados para CLa, CLb e CLc foram de 37,6 e 34,4; 39,6 e 36,9; 41,7 e $37,3 \mathrm{mmol}_{\mathrm{c}} \mathrm{kg}^{-1}$, respectivamente (Figura 2). Comparando-se os valores mínimos de CTC, aos 360 DAIC, com os de T, observa-se que o composto promoveu acréscimos líquidos de carga negativa de 2,7 , 5,1 e 5,6 $\mathrm{mmol}_{\mathrm{c}} \mathrm{kg}^{-1}$ ao final do primeiro ano de aplicação, nos tratamentos CLa, CLb e CLc, respectivamente.

Após a segunda aplicação do composto, ano agrícola 1997/98, embora tenha sido verificado um comportamento quadrático da variável em função das épocas de amostragem (CTC $=40,2-0,02 x E A s+$ $\left.0,00006 \times \mathrm{XAs}^{2}, \mathrm{R}^{2}=0,96, \mathrm{p}<0,01\right)$, não houve decréscimos expressivos na CTC do solo, considerando-se este ano agrícola como um todo (Figura 2). Os valores médios de CTC proporcionados pelos tratamentos CLa, CLb e CLc foram de 38,9 $\pm 0,4 ; 41,9 \pm 0,5$ e $43,8 \pm 0,3 \mathrm{mmol}_{\mathrm{c}} \mathrm{kg}^{-1}$, respectivamente, os quais significaram, quando comparados a $\mathrm{T}$, acréscimos de $7,6 \pm 0,3 ; 10,7 \pm 0,5$ e $12,5 \pm 0,2$ mmol $_{\mathrm{c}} \mathrm{kg}^{-1}$.
Aumentos na CTC de solos pela aplicação de compostos de lixo também foram verificados por Hortenstine \& Rothwell (1972), Bengtson \& Cornette (1973), Giusquiani et al. (1995) e Abreu Junior (1999).

Outro aspecto importante a ser considerado diz respeito à $\mathrm{CTC}$ a pH 7,0 do próprio composto de lixo (Tabela 2). Estimativas de acréscimos da CTC do solo, realizadas em função desses valores, da taxa de aplicação do resíduo e da densidade do solo na camada de incorporação, revelaram acréscimos potenciais, respectivamente, para CLa, CLb e CLc, ao redor de 1,1, 2,2 e 3,3 mmol $_{\mathrm{c}} \mathrm{kg}^{-1}$ em 1996/97 e de 2,1, 4,2 e $6,3 \mathrm{mmol}_{\mathrm{c}} \mathrm{kg}^{-1} \mathrm{em} \mathrm{1997/98}$. Portanto, considerando a CTC a pH 7,0 do composto de lixo, essencialmente, função de sua matéria orgânica, é provável que estes efeitos sobre a CTC a pH atual do solo sejam resultados da interação entre os ganhos de $\mathrm{C}$ orgânico proporcionados ao mesmo e os incrementos no pH causados pela aplicação do composto. Essa hipótese foi comprovada pelas correlações estabelecidas entre CTC x pH e CTC x C orgânico do solo (Tabela 6).

Resultados semelhantes foram obtidos por Rodella et al. (1995) e Rodella (1996) que constataram que o efeito de diversos resíduos orgânicos sobre a CTC a pH atual de solos sob clima tropical está diretamente relacionado com o efeito desses resí-

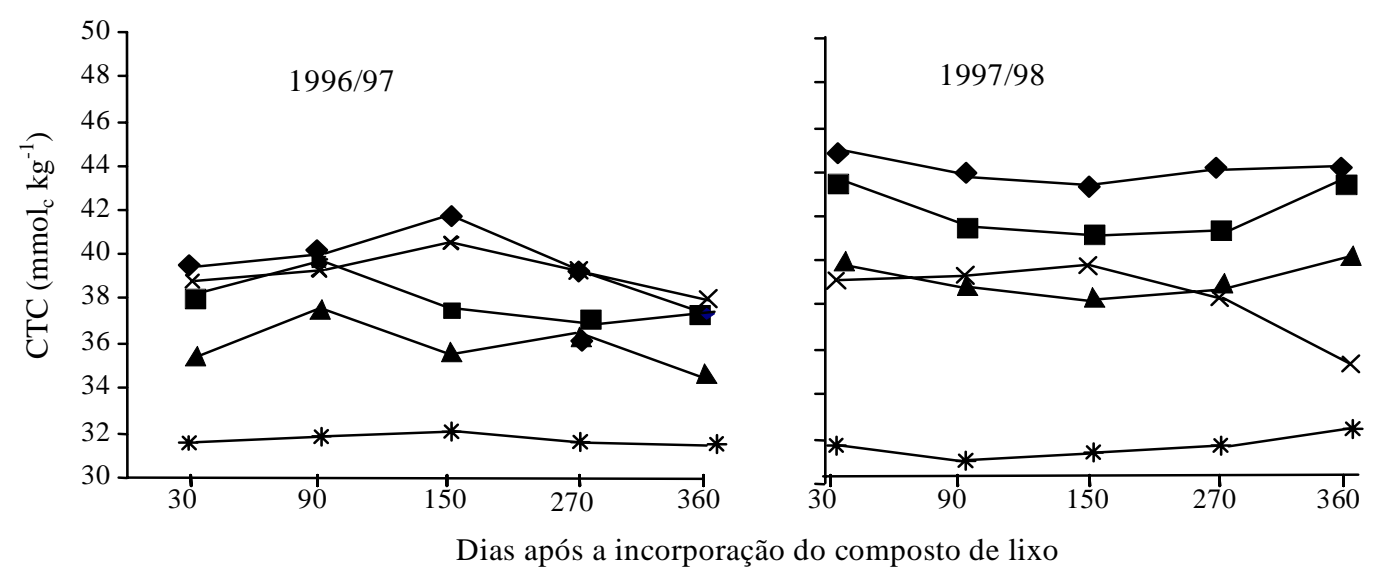

Figura 2. Capacidade de troca de cátions do solo nos anos agrícolas de 1996/97: testemunha absoluta (*); $2 \mathrm{Mg} \mathrm{ha}^{-1}$ de cálcario dolomítico + adubação de plantio $(\times), 20(\mathbf{\Delta}), 40(\square)$ e $60 \mathrm{Mg} \mathrm{ha}^{-1}(\bullet)$ de composto de lixo, e 1997/98: testemunha absoluta (*), adubação de cobertura na primeira soqueira $(X), 24(\mathbf{\Lambda}), 48(\square)$ e $72 \mathrm{Mg} \mathrm{ha}^{-1}(\bullet)$ de composto de lixo. 
duos sobre o pH dos solos. Abreu Junior et al. (2000) verificaram uma relação inversa entre a CTC inicial de solos ácidos e o incremento nos valores de $\mathrm{pH}$, devido à aplicação de $60 \mathrm{Mg} \mathrm{ha}^{-1}$ de composto de lixo de modo que, solos com elevada CTC apresentaram maior capacidade de resistir a mudanças em face do seu elevado poder tampão.

Todavia, não se pode afirmar que os aumentos verificados na CTC sejam exclusivamente devidos à matéria orgânica do composto de lixo. Um indicativo disto é dado pelos resultados apresentados pelo tratamento AM (Figura 2), o qual apresentou valores médios de CTC, durante os dois anos agrícolas, de $38,6 \pm 0,5 \mathrm{mmol}_{\mathrm{c}} \mathrm{kg}^{-1}$ representando um acréscimo em relação à $\mathrm{T}$, de $7,1 \pm 0,5 \mathrm{mmol}_{\mathrm{c}} \mathrm{kg}^{-1}$. Considerando-se que o valor médio de $\mathrm{pH}$ nos dois anos foi de 5,2 no tratamento AM e de 4,2 em T, verifica-se que apenas a elevação do $\mathrm{pH}$ do solo foi capaz de aumentar sua CTC. No primeiro ano, por exemplo, o efeito do tratamento AM sobre a CTC foi superior aos verificados em CLa e CLb, e equivalente ao de CLc (Figura 2).

É importante salientar que a observação desses fenômenos só foi possível pelo fato da CTC ter sido determinada ao $\mathrm{pH}$ atual do solo. Determinações realizadas com soluções alcalinas tamponadas ou por soma de bases certamente iriam mascarar esses resultados.

De maneira geral, os decréscimos da CTC verificados para CLa, CLb e CLc, no transcorrer de 1996/97, podem ser explicados pelos decréscimos do $\mathrm{C}$ orgânico e pH do solo. Por outro lado, em 1997/98 a não ocorrência deste efeito pode ser atribuída à menor taxa de decréscimo do $\mathrm{C}$ orgânico, e principalmente pela manutenção dos valores $\mathrm{pH}$ (Tabelas 3 e 5).

Tabela 6. Coeficientes de correlação linear simples entre valores obtidos para CTC e pH, e CTC e teores carbono orgânico do solo nos anos agrícolas 1996/97 e 1997/98.

\begin{tabular}{lcc}
\hline Ano agrícola & CTC x pH & CTC x C orgânico \\
\hline $1996 / 97$ & $0,73^{* * *}$ & $0,74^{* * *}$ \\
$1997 / 98$ & $0,91^{* *}$ & $0,89^{* *}$ \\
$1996 / 97$ e 1997/98 & $0,85^{* *}$ & $0,84^{* *}$ \\
\hline
\end{tabular}

${ }^{* *}$ Significativo a $1 \%$ de probabilidade, pelo teste $t$.

\section{Conclusões}

1. Aplicações anuais e sucessivas de composto de lixo, em doses superiores a $20 \mathrm{Mg} \mathrm{ha}^{-1}$, aumentam os teores de carbono orgânico, a condutividade elétrica e os valores de $\mathrm{pH}$ e CTC a pH atual do solo.

2. A condutividade elétrica do solo, em áreas tratadas com composto de lixo, deve ser monitorada para se evitar possíveis efeitos salinos sazonais.

\section{Referências}

ABREU JUNIOR, C. H. Propriedades químicas e disponibilidade de nutrientes e de metais em diferentes solos adubados com composto de resíduo urbano. 1999. 159 f. Tese (Doutorado) - Escola Superior de Agricultura Luiz de Queiroz, Piracicaba. 1999.

ABREU JUNIOR, C. H.; MURAOKA, T.; LAVORANTE, A. F.; ALVAREZ, V. F. C. Condutividade elétrica, reação do solo e acidez potencial em solos adubados com composto lixo. Revista Brasileira de Ciência do Solo, Viçosa, v. 24, n. 3, p. 645-657, 2000.

BARRETO, M. C. V. Degradação da fração orgânica de diferentes resíduos e efeitos em algumas propriedades químicas e físicas de dois solos. 1995. $106 \mathrm{f}$. Tese (Doutorado) - Escola Superior de Agricultura Luiz de Queiroz, Piracicaba. 1995.

BEngtson, G. W.; CORNETTE, J. J. Disposal of composted municipal waste in a plantation of young slash pine: effects on soil and trees. Journal of Environmental Quality, Madison, v. 2, n. 4, p. 441-444, 1973.

BENITES, V. M.; MENDONÇA, E. S. Propriedades eletroquímicas de um solo eletropositivo influenciadas pela adição de matéria orgânica. Revista Brasileira de Ciência do Solo, Viçosa, v. 22, p. 215-221, 1998.

BERNAL, M. P.; SÁNCHES-MONEDERO, M. A.; PAREDES, C.; ROIG, A. Carbon mineralization from organic wastes at different composting stages during their incubation with soil. Agriculture, Ecosystems and Environment, Amsterdam, v. 69, p. 175-189, 1998.

EATON, A. D.; CLESCERI, L. S.; GRENNBERG, A. E. Standard methods for the examination of water and wastewater. 19. ed. Washington: American Public Health 
Association/American Water Works Association/Water Environment Federation, 1995. 1082 p.

GILLMAN, G. P.; SUMPTER, E. A. Modification to the compulsive exchange method for measuring exchange characteristics of soils. Australian Journal of Soil Research, Melbourne, v. 24, p. 61-66, 1986.

GIUSQUIANI, P. L.; PAGLIAI, M.; GIGLIOTTI, G.; BUSINELLI, D.; BENETTI, A. Urban waste compost: effects on physical, chemical, and biochemical soil properties. Journal of Environmental Quality, Madison, v. 24, n. 1, p. 175-182, 1995.

HARDING, S. A.; CLAPP, C. E.; LARSON, W. E. Nitrogen availability and uptake from field soils five years after addition of sewage sludge. Journal of Environmental Quality, Madison, v. 14, n. 1, p. 95-100, 1985.

HORTENSTINE, C. C.; ROTHWELL, D. F. Pelletized municipal refuse compost as a soil amendment and nutrients source for sorghum. Journal of Environmental Quality, Madison, v. 2, n. 3, p. 343-345, 1973.

HORTENSTINE, C. C.; ROTHWELL, D. F. Use of municipal compost in reclamation of phosphate-mining sand tailings. Journal of Environmental Quality, Madison, v. 1, n. 4, p. 415-418, 1972.

HOYT, P. B.; TURNER, R. C. Effects of organic materials added to very acid soil on $\mathrm{pH}$, aluminium, exchangeable $\mathrm{NH}_{4}$ and crop yields. Soil Science, New Brunswick, v. 119 , p. $227-237,1975$.

HUE, N. V. Correcting soil acidity of a highly weathered ultisol with chicken manure and sewage sludge. Communications in Soil Science and Plant Analysis, New York, v. 23, n. 3/4, p. 241-264, 1992.

LEVI-MINZI, R.; RIFFALDI, R.; SAVIOZZI, A. Carbon mineralization in soil amended with different organic materials. Agriculture, Ecosystems and Environment, Amsterdam, v. 31, p. 325-335, 1990.

MATTIAZZO, M. E.; GLÓRIA, N. A. Effect of vinasse on soil acidity. Water Science and Technology, Oxford, v. 19, n. 7, p. 1293-1296, 1987.
OLIVEIRA, F. C. Disposição de lodo de esgoto e composto de lixo urbano num Latossolo Vermelho-Amarelo cultivado com cana-de-açúcar. $2000.247 \mathrm{f}$. Tese (Doutorado) - Escola Superior de Agricultura Luiz de Queiroz, Piracicaba. 2000.

PASCUAL, J. A.; HERNANDEZ, T.; GARCIA, C.; AYUSO, M. Carbon mineralization in an arid soil amended with organic wastes of varying degrees of stability. Communications in Soil Science and Plant Analysis, New York, v. 29, n. 7/8, p. 835-846, 1998.

RODELLA, A. A. Métodos de avaliação de materiais orgânicos e efeitos de sua incorporação ao solo sobre a mobilização de macronutrientes. 1996. $148 \mathrm{f}$. Tese (Livre Docência) - Escola Superior de Agricultura Luiz de Queiroz, Piracicaba. 1996.

RODELLA, A. A.; FISCHER, K. R.; ALCARDE, J. C. Cation exchange capacity of an acid soil as influenced by different sources of organic matter. Communications in Soil Science and Plant Analysis, New York, v. 26, n. 17/18, p. 2961-2967, 1995.

SPIRONELLO, A.; RAIJ, B. van.; PENATTI, C. P.; CANTARELLA, H.; MORELLI, J. L.; ORLANDO FILHO, J.; LANDELL, M. G. A.; ROSSETO, R. Cana-de-açúcar. In: RAIJ, B. van.; CANTARELLA, H.; QUAGGIO, J. A.; FURLANI, A. M. C. (Ed.). Recomendações de adubação e calagem para o Estado de São Paulo. Campinas: IAC, 1996. p. 237-239.

TRINDADE, A. V.; VILDOSO, C. I. A.; MUCHOVEJ, R. M. C.; COSTA, L. M. Interação de composto de lixo urbano e fungos micorrízicos na nutrição e crescimento do milho. Revista Brasileira de Ciência do Solo, Viçosa, v. 20, n. 2, p. 199-208, 1996.

WONG, M. T. F.; NORTCLIFF, S.; SWIFT, R. S. Method for determining the acid ameliorating capacity of plant residue compost, urban waste compost, farmyard manure, and peat applied to tropical soils. Communications in Soil Science and Plant Analysis, New York, v. 29, p. 2927-2937, 1998

XIN, T. H.; TRAINA, S. J.; LOGAN, T. J. Chemical properties of municipal solid waste compost. Journal of Environmental Quality, Madison, v. 21, n. 3, p. 318-329, 1992. 